\title{
Automated Diabetic Retinopathy Identification Using Convolutional Neural Network
}

\author{
Vidya Manjunath Purushan ${ }^{1}$, Dr. Manja Naik.. ${ }^{2}$ \\ ${ }^{1}$ PG Scholar, Department of Electronics and Communication, UBDT College of Engineering, India, \\ vidyampec@gmail.com \\ ${ }^{2}$ Professor, Department of Electronics and Communication, UBDT College of Engineering, India, \\ manjubdt2009@gmail.com
}

\begin{abstract}
Classification phase is one of the important step for determining, analysing as well as diagnosing the diabetic retinopathy disorder. Nanostructures include red lesions, retinal hard macular exudates as well as Neovascularization would take up space around retina by the reason of devastation of veins. In order to computerise the technique pertaining to diabetic retinopathy phases categorization, a convolution neural network grounded method could be utilized. Colour fundus pictures of retina are collected during this work with aim of diabetic retinopathy classification among 5 phases by utilizing a convolution neural network. Convolution neural network with EfficientNet B5 network is employed for the phase classification of diabetic retinopathy disorder, a Kappa value (classification accuracy) of $88.48 \%$ is achieved.
\end{abstract}

Key words: Convolution neural network $(\mathrm{CNN})$, Diabetic retinopathy, EfficientNet B5.

\section{INTRODUCTION}

Persistence diabetes mellitus causes the optic disorder is Diabetic Retinopathy. This ailment has bad impact on tiny met arteriole and Retinal Neuron and also causes severe harm. This give rise to seepage in vein of eye and protuberance, blocks the blood there by avert it to pass over along with this, increment of anomalous unfamiliar vein of retina occurs occasionally. Concatenation of blemish around eyesight, weakened trichromats, differ in eyesight, vacant region around sight, unfocused sight together with deprivation of sight are complete features of this disorder. There are numerous methods are available in order to manage the diabetic retinopathy, even so these are not at all a proper heal. Succeeding optic nerve destruction along with sight loss feasible as well because it is deep-rooted and persistent disorder. Consequently, a decent recognition of disorder is obligatory. So for that purpose, an automated method gives an instant result in forecasting disorder is necessary which is better agreeable by surgeon along with sick person as well. Therapy for the disorder works well if it is recognized in initial phase. Convolution Neural Network comes under the group of Deep Neural Network in deep learning, brings solution in determining disorder at early stage.
Table 1: Different stages of Diabetic Retinopathy.

\begin{tabular}{|c|c|c|}
\hline Stage & $\begin{array}{l}\text { Dilated Ophthalmoscopy Observable } \\
\text { Findings }\end{array}$ & Severity \\
\hline I & No abnormalities & No DR \\
\hline ॥ & Micro-aneurysms only & $\begin{array}{l}\text { Mild non- } \\
\text { proliferative } \\
\text { DR }\end{array}$ \\
\hline III & $\begin{array}{l}\text { Any of the following: } \\
\text { - micro-aneurysms } \\
\text { - retinal dot and blot haemorrhages } \\
\text { - hard exudates or cotton wool spots } \\
\text { No signs of severe non-proliferative } \\
\text { diabetic retinopathy }\end{array}$ & $\begin{array}{c}\text { Moderate } \\
\text { non- } \\
\text { proliferative } \\
\text { DR }\end{array}$ \\
\hline IV & $\begin{array}{l}\text { Any of the following: } \\
\text { - more than } 20 \text { intra-retinal } \\
\text { hemorrhages in each of } 4 \text { quadrants } \\
\text { - definite venous beading in } 2 \text { or more } \\
\text { quadrants } \\
\text { - prominent intra-retinal microvascular } \\
\text { abnormality (IRMA) in } 1 \text { or more } \\
\text { quadrants } \\
\text { No signs of proliferative retinopathy }\end{array}$ & $\begin{array}{l}\text { Severe non- } \\
\text { proliferative } \\
\text { DR }\end{array}$ \\
\hline v & $\begin{array}{l}\text { One or both of the following: } \\
\text { - Neovascularization } \\
\text { - Vitreous/pre-retinal hemorrhage }\end{array}$ & $\begin{array}{l}\text { Proliferative } \\
\text { DR }\end{array}$ \\
\hline
\end{tabular}

Convolution Neural Network generally used for perceptible imagery inspection. It is a model which is more normalized and contains many layers' perceptron. This symbolize that CNN has Networks which is joined fully. Neuron which belongs to that particular layer is linked among each and every neurons of other layer next to it. Due to this type of connectivity leads to high variance of data. By adjusting the weights evokes reduction in loss thus high variance of data gets normalized. Motivation behind CNN is came from procedures of biological. Approximately a small portion of pre-processing utilized by CNN in contrast with another algorithm utilized for classification of images. This pave the way for the enhancement of filter, done by the network after grasping. The ability of selfgoverned from advance learning along with person involution during characteristic extirpation is extensive supremacy of CNN. 

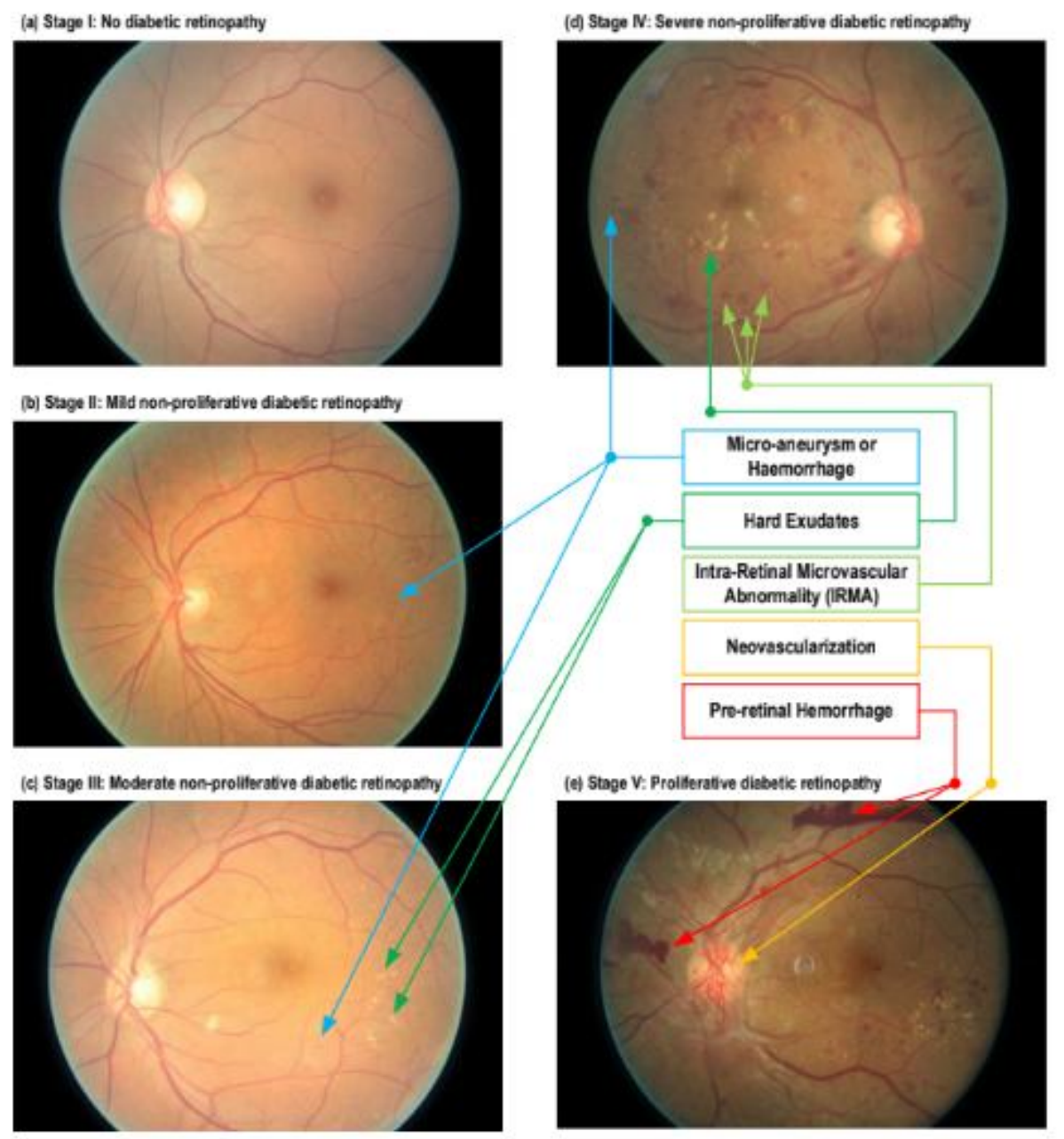

Figure1: Fundoscopic images of various phases of diabetic retinopathy. (a)Phase1: No diabetic retinopathy(Normal); (b)Phase2: Mild nonproliferative diabetic retinopathy; (c)Phase3: Moderate non-proliferative diabetic retinopathy;(d) Phase4: Severe non-proliferative diabetic retinopathy; (e)Phase5: Proliferative diabetic retinopathy.

Generally, CNN's established by a stable resource amount along with, later increased production capacity for the purpose of achieving superior precision once the extra resource is provided. EfficientNet is one such an architecture used to improve the precision of model, which is built by CNN. The potency included in design measuring moreover depend upon Network baselining. This architecture attains preferred precision along with effectiveness in contrast with other current ImageNet using $\mathrm{CNN}$.

Extensive reason behind sightlessness in diabetic patient is due to diabetic retinopathy disorder. Recognizing this disorder at initial level is very complicated. So the prototype developed by using Convolution Neural Network along with EfficientNetB5 architecture together with transfer of learning methodology. The presented methodology receives images of fundus being input as well as ascertained their interrelationship in order to build a better precision.

In order to do the retinal development tracking, photography of fundus is utilized. Identical retinal area anatomical structure via distinct angles by equivalent moment as well as it is utilized to reach rebuilding of fundus into three dimension.

\section{LITERATURE SURVEY}

Screening test for the purpose of DR disorder made the CNN's to put into set of rules by the clinician during this modern era. Images feed in to the network as a input, those images convolute accompanied by definitive weighing matrix. This helps to take out distinct attribute of images with no ruining of spacing details. Distinct architectonics were estimated at first in order to decide the finest CNN which suit outstanding so as to achieve classification of Binary. Multinomial classification design was trained with a view to magnify the phases sensitiveness of Retinopathy like premature phase levels [4]. Followed by this is coupled with cleaning of data together with Augmentation of dataset. These steps helps to boost precision of test data in addition to hike valid package of data size of sample. Directed care as regard to obedience of data as well as standard after gathering images which are validated by optometric physician. Directed the problem of inadequate amount of observation that put into study while utilizing deep convolution neural network including transfer of training covering colour feature for the functional learning discriminant in order to do the identification work. Utilized both AlexNet along with GoogleNet CNN architectonics for training as well as testing purpose. 
Make use of CNN which is already the trained one brings the prospective advantage of categorizing a huge number of images at all time. This makes it to employ for a live application at any time we obtain a current image. Usually images delivered to specialist in case of reality in order to classify it but inexact in classifying that image while sufferer is put for test in a systematic way, because it is even difficult to classify for experienced specialist. Convolution neural network which is built after training gives a immediate examination including feedback towards sufferer feasible. This Neural Network have the capacity of examination of image in a magnificent way. It even work well for the multiplex image identification issues, because of its enlarge calculation ability via GPU, RELU activation function, along with withdraw application [3]. The couple of problem occur inside CNN along with while computerized classifying. Those problem includes attaining advantageous counterweight featuring in sufferer who accurately recognized possessing disorder called sensitivity along with sufferer who accurately recognized without diabetic retinopathy called specificity. Such a problem is remarkably difficult being 5 category problem like NO DR, mild NDPR, moderate NDPR, severe NDPR along with proliferative. Another problem include overfitting. This problem occurs due to group of data which is asymmetrical in nature. If the data contain below 3\% of images accrue deriving out of fourth together with fifth class, signifies the requirement in modification toward neural network so that it might possibly grasp those images characteristics.

ConvolutionNeural Network which is a branch of deep learning is a beneficial methodology toward entire phases of retinopathy disorder in diabetic patient. Amplification of data involves dissimilarity adaptation, cartwheeling of images, glazing alteration [2]. Local data of eye fundus usually used by deep CNN networks. Initial foot-pace in cleaning of data followed by sizing up the images. Change the images toward black and white or representing colour with shades of gray previous to delivering it to architectonics afterwards change it to $\mathrm{L}$ design. In order to accentuate the microaneurysms along with vein of eye in the images. For the purpose of identification of images $\mathrm{CNN}$ network is utilized.

One of the class of neural networks is CNNs, these networks showed more successful in identification of image along with categorization. This paper mainly focusses on 5 stages of diabetic retinopathy identification by utilizing InceptionNet along with VGG16 architectures in order to build CNN. ReLU activation function is preferred because in order to make all negative values element in images as zero. In order to keep only main features along with to bring down dimensionality, pooling layer is preferred. About transfer learning and to carry out it effectively without misplace its constancy, images can be rescaled to $227 * 227$ or $224 * 224$ or $299 * 299$ pixels along with advantageous of utilizing stochastic gradient descent also discussed in this paper [5].

Displayedtheunimpaired vs impaired retinal due to diabetic retinopathy by utilizing vision algorithms for embedded vision. Such a innovation possess a power to actively recognize diabetes retinopathy disorder by utilizing this algorithm. This algorithm recognizes all the 5 phases of disorder. The construction of convolution neural network involves layers of pooling together with convolution. The key overlap of this network is convolution layer for the purpose of achieving convolution activity along with this, in order to lower the dimensionality pooling overlap is used [6]. Followed by this the overlap which linked fully is utilized to categorize impaired and unimpaired retina. Tensorflow, a non-proprietary library, provides all time result meant for artificial intelligence. At last model which is built is loaded and experimented for test images.

The motivation behind the man-made neural network came from biological processes of our brain. The mechanism of this manmade neural network is equivalent to human neural system in a way how our brain mechanizes the information. CNN which is deep neural network perform well in a precise manner for the categorization of image. The projected design by utilizing deep $\mathrm{CNN}$ architecture is going to be used in machinery that is linked to fundus camera [7].

The three training algorithms like AlexNet, VGG16, Inceptionnet V3 [1] were designed and their performance accuracies were evaluated. After that, characteristic arising out of these three models were chained in order to get superior implementation. The already trained designs were adjusted such that tuned up by ImageNet in order to achieve prosperous in training of three neural networks of CNN. Tuned up methods established on the idea pertaining to transfer learning. The models which built by using different architectures can be saved and loaded to in order to test the result for individual images and we can get instant results.

In order to do the classification and there by predict the phases of diabetic retinopathy a deep convolution neural network which is built by eighteen layers of convolution along with three layers which are densely linked is utilized. The presented design here is the improved kind of VGG-19 architecture [10].

Many attribute together with position of those attribute in an image is weighed in order to do the categorization of diabetic retinopathy disorder. CNN neural network which comes under deep neural network is used to provide immediate result. Extremely compounded image identification work along with thing categorization auspiciously done by utilizing CNN. Super Vector Machine [11] categorization algorithm is utilized for the characteristic uproot from images.

\section{ARCHITECTURE AND METHODOLOGY}

\section{A. Primary Knowledge}

One of architectonics of Artificial neural network is convolution neural network mainly utilized for image categorization. To the common Neural network, this convolution neural network put on several additional functions such as nonlinearities, under sampling, convolution. $\mathrm{CNN}$ is divided into two portions. Primary portion being a characteristic uprooting and secondary portion is classification. 


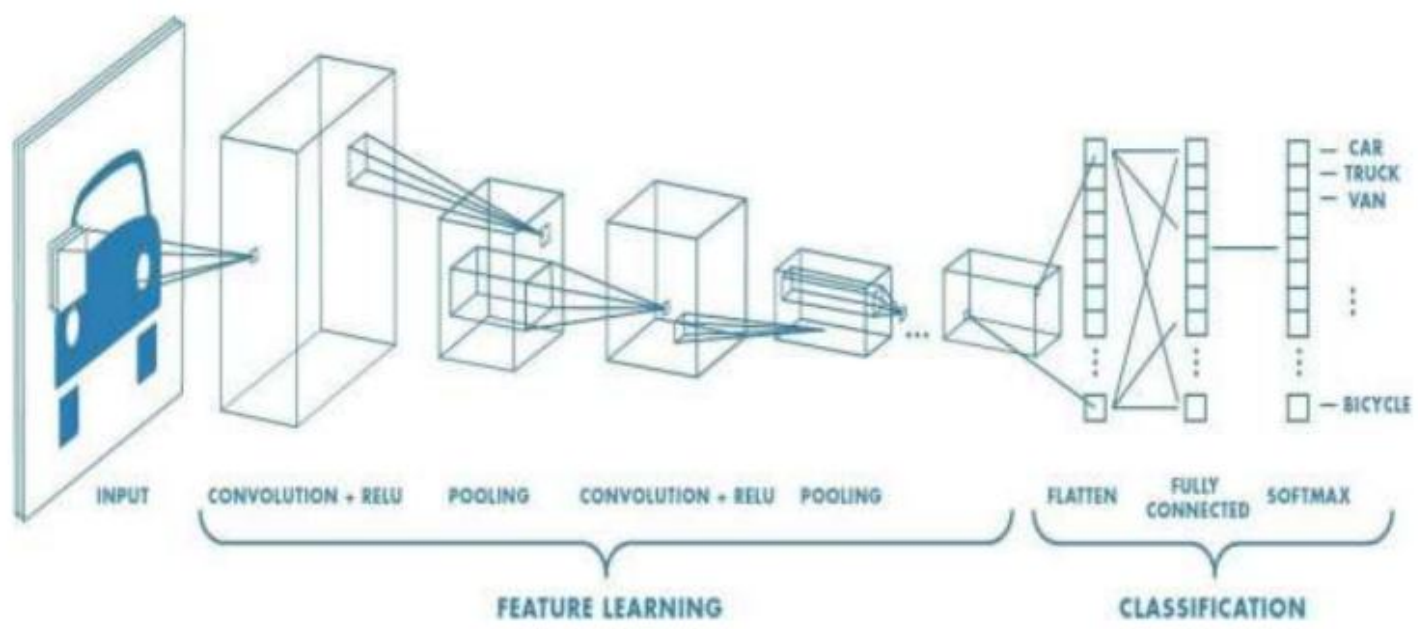

Figure 2: Architecture of Convolution Neural Network.

For the feature determination a sequence of convolution as well as pooling task are executed during primary portion Convolution functioning is practiced so as to achieve characteristic chart by utilizing a filter. This feature chart consists of negative numbers. So these numbers must be substituted by zero. For this purpose a RELU activation function is utilized. This process is also called Non-linearity. Addition to feature uprooting there is a classification portion in which the layers which is fully connected perform classifier. Those classifiers allocate possibility toward image objects. The too broad images measurements are lowered by pooling functioning. Such a functioning helps to lower a lot of calculations complexity as well as variable of neural network. This in turn decreases time required for training as well as overfitting is regulated. Very essential details preserved by Pooling Average, Max as well as Global pooling are different kind of pooling.

\section{B. Architecture of EfficientNet B5}

Efficientnet is main tool toward images of clinical. Google AI emancipated Efficient net architecture in 2019. It is a recently emancipated a unique modernity upon ImageNet. These architectonics utilizes a methodical procedure for convolution neural network scaling almost in excellent method. Here in our project we are using B5 version, for the kernel we had chosen. Such a chosen kernel here, contribute weights to Efficientnet B0 via B5. Google Assistant depository owing to EfficientNet contains EfficientNet B6 as well as EfficientNet B7 weights.

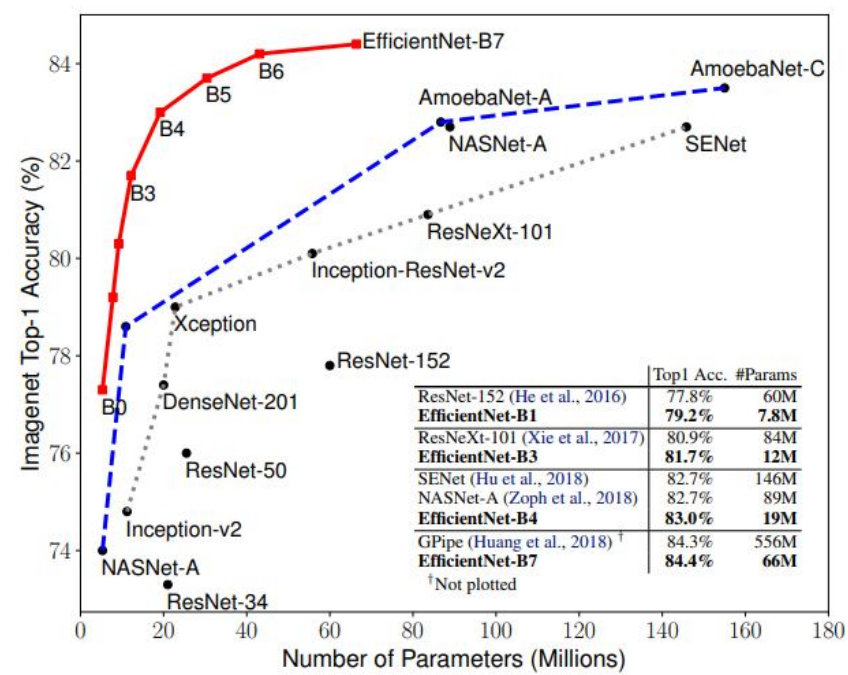

Figure3: Model size vs ImageNet precision.

\section{Generic things in every model:}

Generic thing in whatever the network is stem belonging to it, followed by entire researching accompanied by the architecture begins, as this is generic thing among 8 models of efficientnet from B0 to B7 along with for the last layers. Subsequently, everyone among them consist of seven blocks. Those blocks have subdivision, of which numeral rises as we proceed starting from B0 to B7 efficient models. 


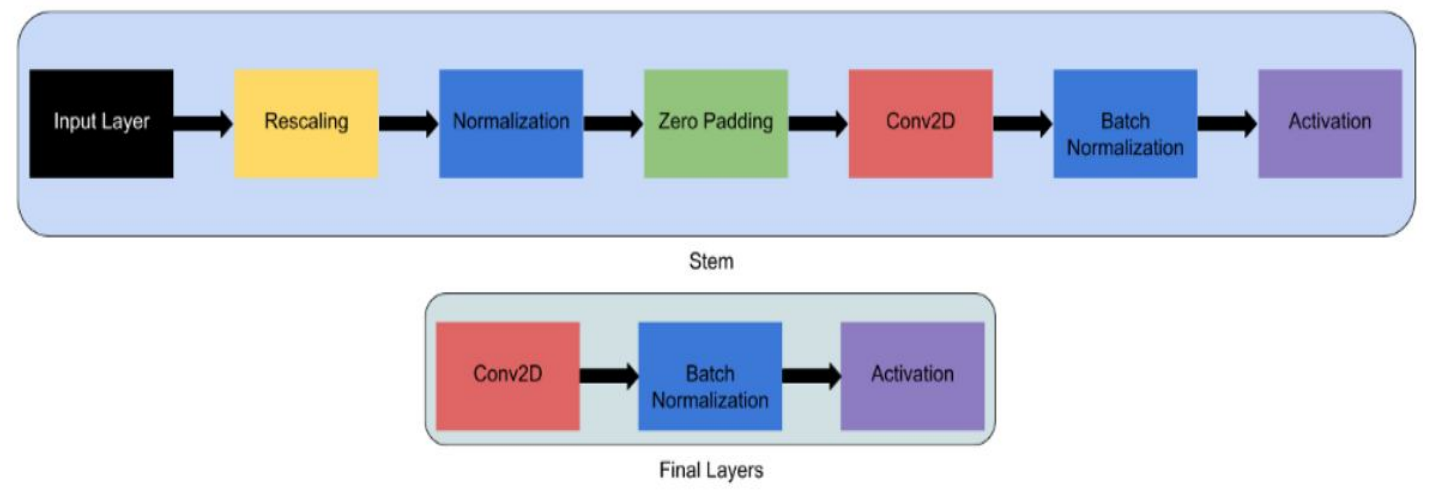

Figure4: Common stem and final layers among 8 models of EfficientNet B5.

Number layers in $\mathrm{B} 0=237$ and in $\mathrm{B} 7$ it is 813 . But whatever be the number, all the layers of the model B0 to B7 built from five modules as displayed in fig beneath as well as stem overhead.

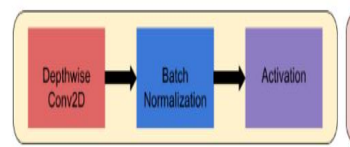

Module 1
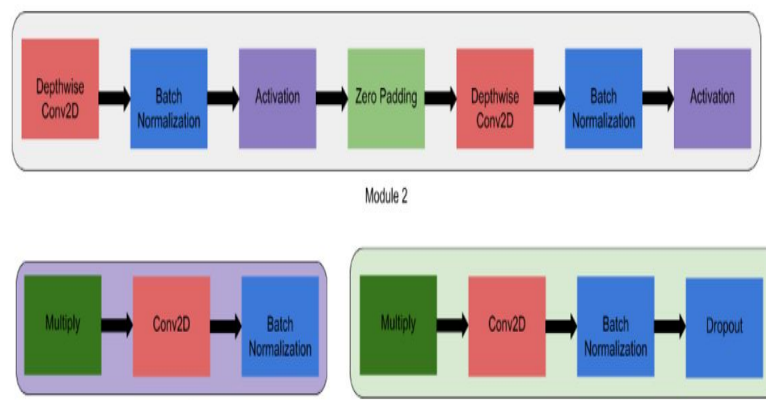

Motule 4
Module 2

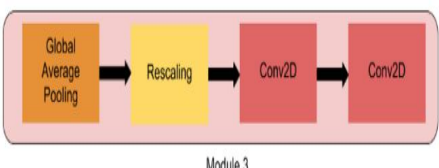

Nodue 3

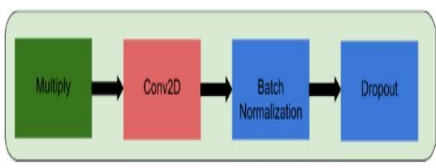

Module 5
Figure5: Utilize all the 5 modules to build architecture.

Module1: Being initial position owing to substitute blocks.

Module2: Out of seven basic block excluding the first, this module is utilized as initial position of first substitute block. Module3: The module-3 attached in act of shortcuts (connection through jumping a few layers) in every sub-blocks.

Module4: Merging shortcuts in first sub-block.

Module5: Individual substitute block is attached toward preceding sub-block in a shortcuts link as well as they are merged by utilizing module 5 .

Those modules merged to configure it to sub-blocks, those can be utilized in a more valid form inside blocks.

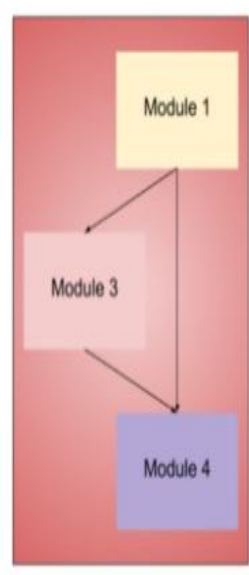

Sub-block 1

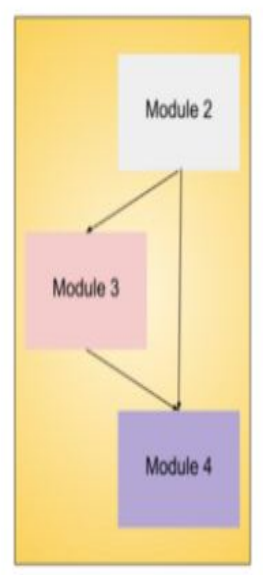

Sub-block 2

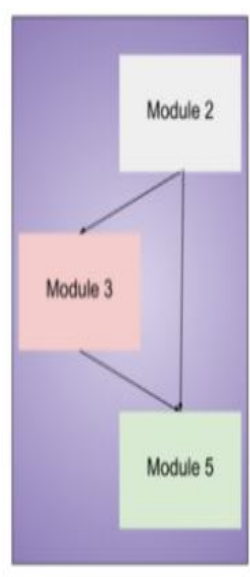

Sub-block 3
Figure6: Sub-blocks of EfficientNet models.

Each one thing discussed until now are merged to build EfficientNet model.

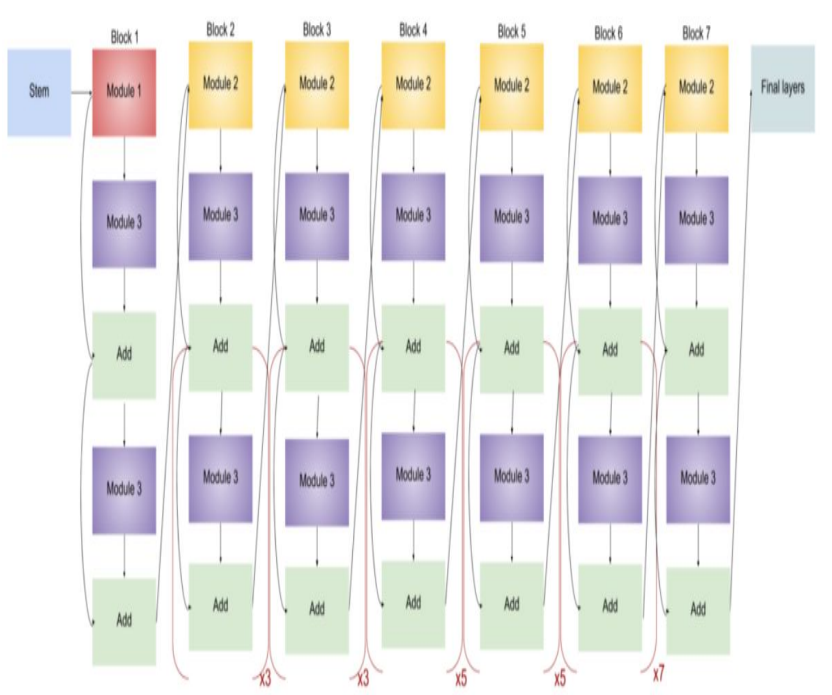

Figure7: Architecture of EfficientNet B5 


\section{BLOCK DIAGRAM}

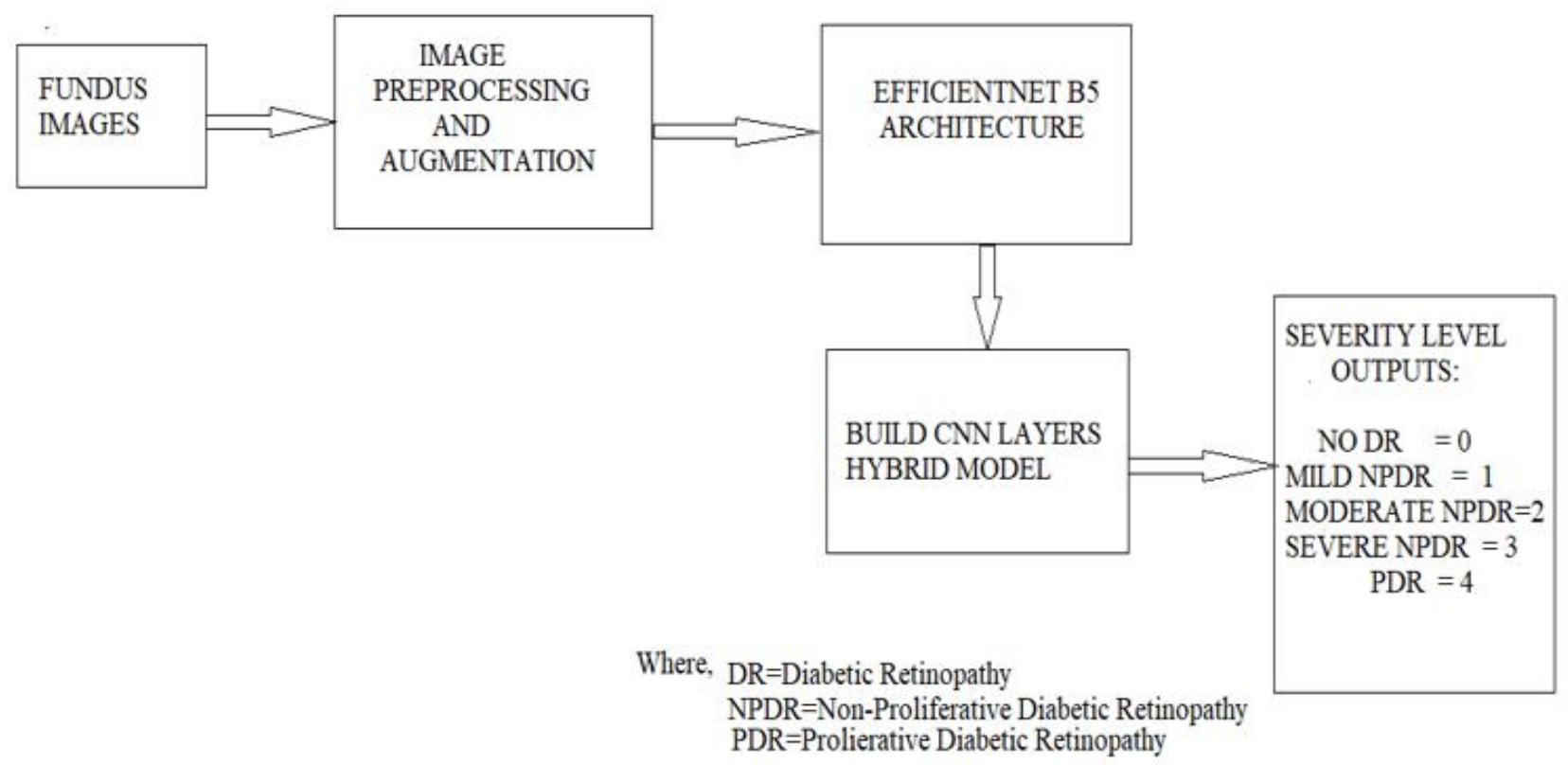

Figure8: Block diagram of Proposed Methodology

The Proposed Methodology contain EfficientNet B5 architecture. We preferred this EfficientNet B5 network being the foundation of convolution neural network. A deep structured learning method together with EfficientNet B5 architecture was employed in order to achieve eye's retina fundus picture classification.

The EfficientNet B5 network grouped the identical scattered buds to form a dense composition in order to expand deepness as well as wideness pertaining to network together with lessen the calculation procedure successfully. This Network deriving out of several had being trained upon ImageNet database accompanied by well-known ground control grades, that potentially will recognize thousands of classifications.

During training procedure, the PEL (Picture Element) intensity of every images as well as related familiar grades were given as input to the network in order that variables within the network gets adapted accordingly to produce a great precision forecast derived from the performance erroneous determined by subtracting among produced model output (predicted value) as well as observed value. For all selected image, the above indicated procedure can be reiterated several times as far as we get perfectly trained network.
In the starting, we set the convolution layers accompanied by weights of prior trained, those were primarily computed out of ImageNet database along with reserved for the purpose of quicken the training operation as well as to bring down redundant. The final layer of EfficientNet B5 network called as fully connected layers were assigned with arbitrary weights with aim that inequitable characteristics field move against ImageNet pictures toward retina of eye fundus pictures might be knowledgeable. The final layer which is modified into 5 classification severity level output as NO DR $=0$, Mild NPDR $=1$, Moderate $\mathrm{NPDR}=2$, Severe $\mathrm{NPDR}=3$ as well as $\mathrm{PDR}=4$ rather than thousands of output regarding ImageNet.

After that entire weight variables pertaining to convolution layers as well as analogous Max pooling layer were frosted for the purpose of bring out the characteristics applicable for the diabetic retinopathy disorder recognition. This implies that firm weights won't modified whereas the arbitrary weights should be bringing up to date such that refreshed throughout training phase. 


\section{FLOW CHART}

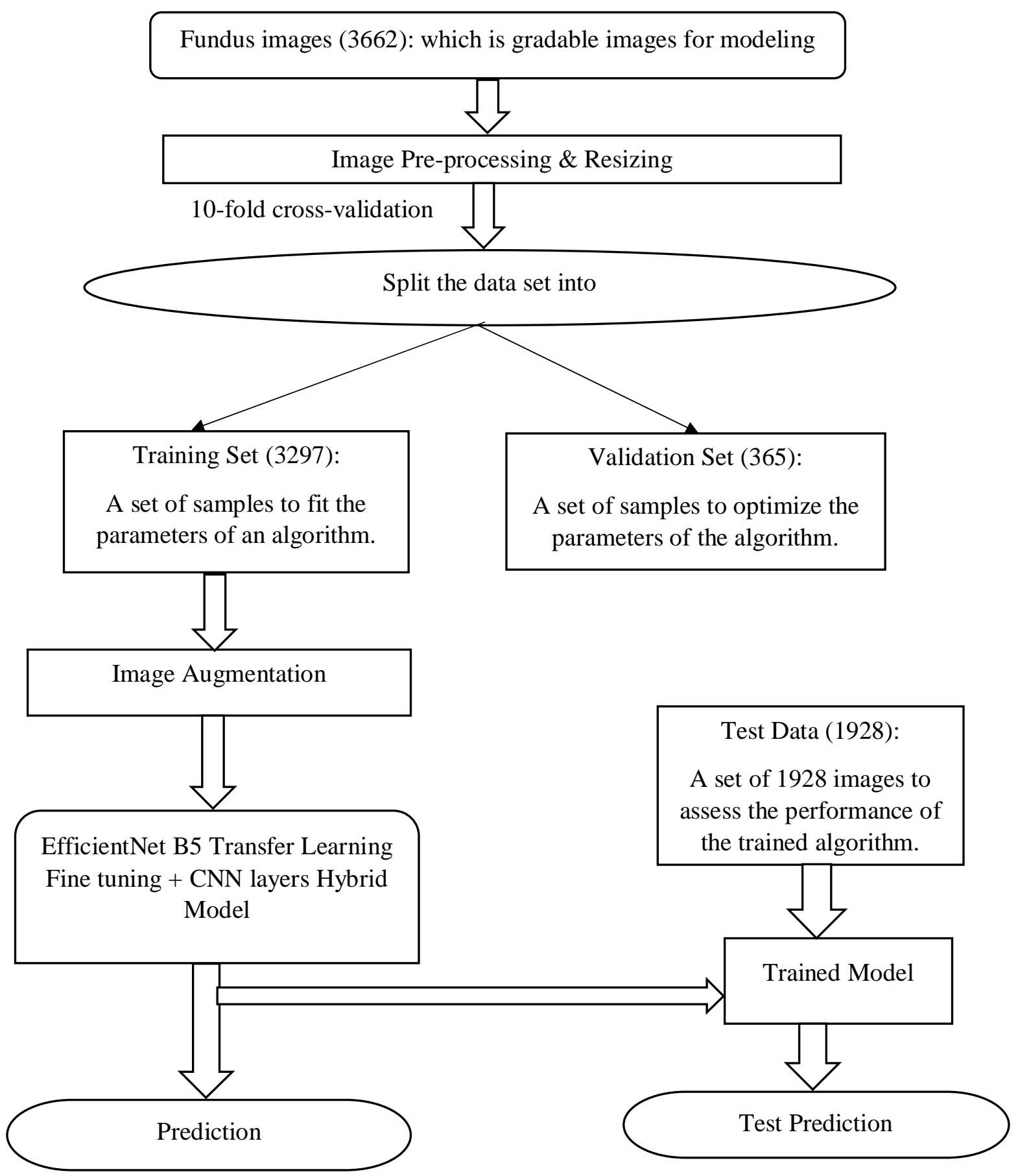

Figure 9: Flowchart of Proposed Methodology

\section{RESULTS AND DISCUSSION}

The fundus retinal images were obtained and separated as training and validation data set in a 9:1 proportion inside each fold after performing 10 fold-cross validations. Training images were trained during training stage to build a model. Once the model is built, the model is saved in a .h5 extension.

The below shown fig is a training images data file containing 3662 images, which is feed as input to the machine in order to build a model and saved as .h5 format. 


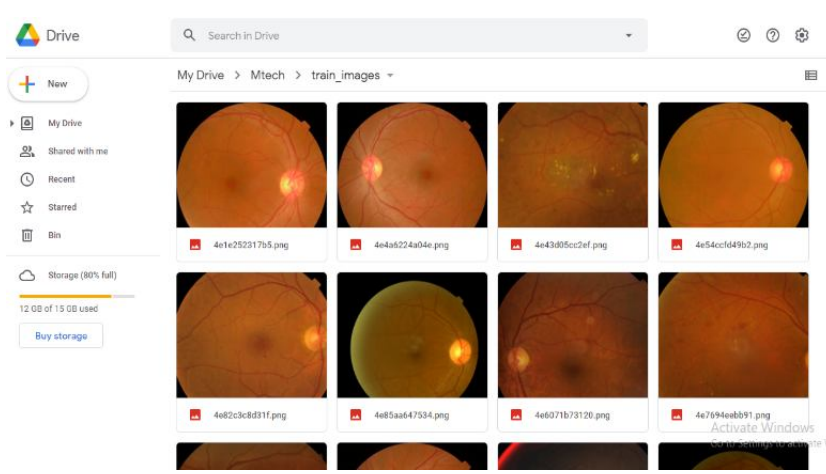

Figure10: Training images data file.

Those training images are images which are graded and labelled by the ophthalmologist into 5 classes. Those are NORMAL $=0$, MILD NPDR $=1$, MODERATE NPDR=2, SEVERE NPDR=3, POLIFERATIVE $=4$. After grading these images fed to the machine.

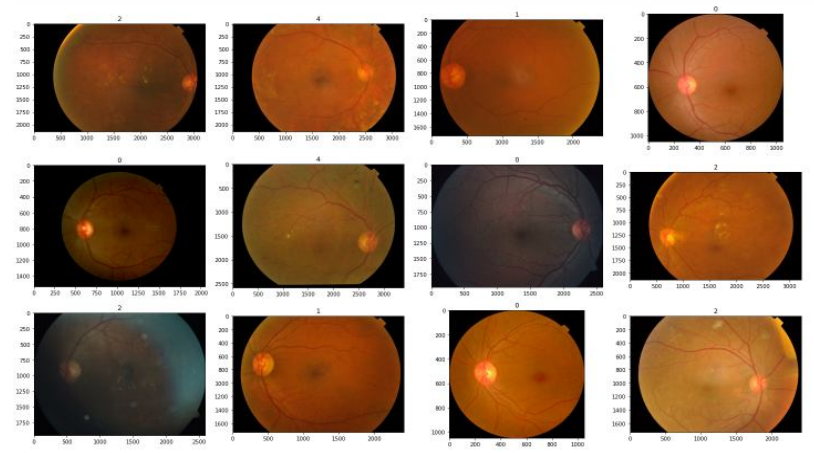

Figure11: Graded training images which contain all five classes.
Table2: Comparison of several features among models built using Inception V3 network and our proposed EfficientNet-B5 network. We can note here that EfficientNet-B5 is finest choice in matter of trainable parameters as well as Kappa value (a classification accuracy).

\begin{tabular}{|l|l|l|}
\hline $\begin{array}{l}\text { Evaluation } \\
\text { Parameter }\end{array}$ & $\begin{array}{l}\text { EfficientNet-B5 } \\
\text { (Proposed) }\end{array}$ & Inception V3 \\
\hline Total Parameters & $29,104,113$ & $22,393,377$ \\
\hline $\begin{array}{l}\text { Trainable } \\
\text { Parameters }\end{array}$ & $28,931,377$ & $22,358,945$ \\
\hline $\begin{array}{l}\text { Non-trainable } \\
\text { Parameters }\end{array}$ & 172,736 & 34,432 \\
\hline Kappa Value & $88.48 \%$ & $83.22 \%$ \\
\hline
\end{tabular}

Graphical comparison of Kappa value (classification accuracy) among Proposed model using Efficientnet B5 and Existing model. Ref no: [12] is shown in below graphs:

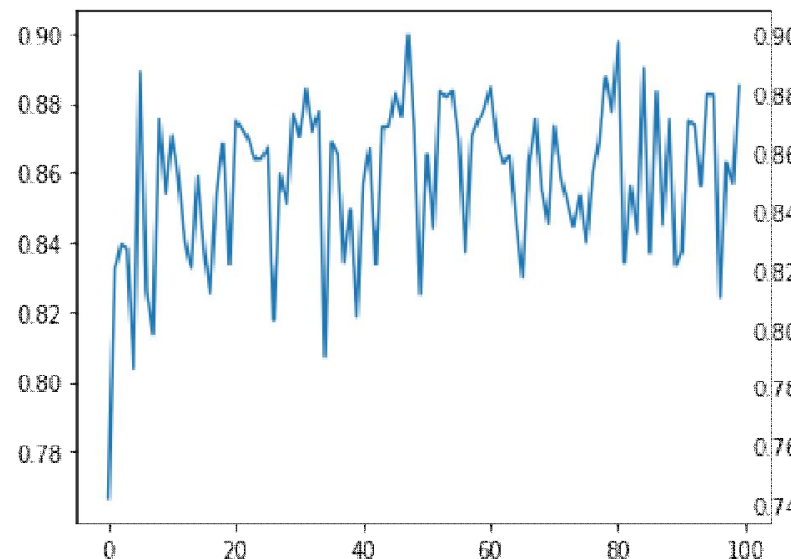

(a)

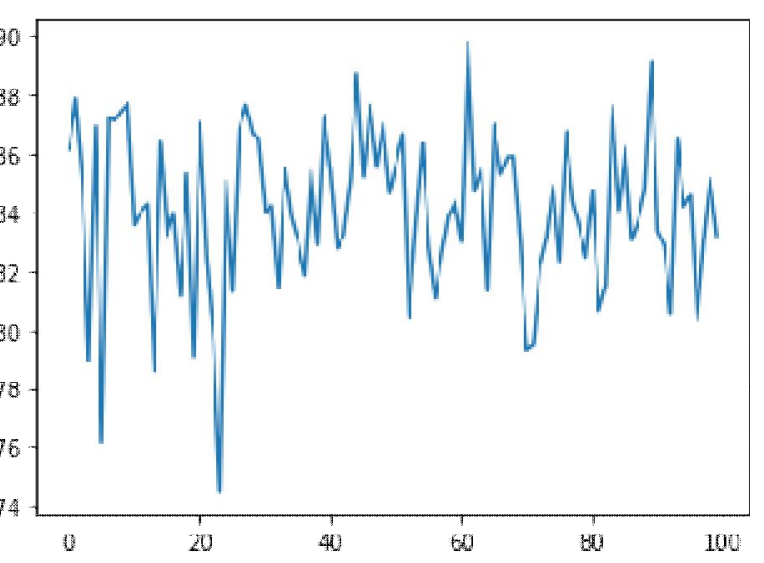

(b)

Figure12: Epoch vs Kappa value of the Proposed and existing methodology. (a) Proposed methodology using EfficientNet B5 with a Kappa value (classification accuracy) of 88.48\% at 100 epoch. (b) Existing Methodology using Inception V3 with a Kappa value of $83.22 \%$ at 100 epoch. 
Table3: Confusion matrix outcome is shown the Classification details pertaining to the dataset which are trained already.

\begin{tabular}{|c|c|c|c|c|c|}
\hline \multirow{3}{*}{$\begin{array}{l}\text { Actual } \\
\text { Results }\end{array}$} & \multicolumn{5}{|c|}{ Predicted Results } \\
\cline { 2 - 6 } & 0-No DR & 1-Mild & 2-Moderate & 3-Severe & 4-Poliferative DR \\
\cline { 2 - 6 } & 179 & 1 & 0 & 0 & 0 \\
\cline { 2 - 6 } & 4 & 27 & 4 & 2 & 0 \\
\cline { 2 - 6 } & 0 & 25 & 67 & 5 & 0 \\
\cline { 2 - 6 } & 0 & 0 & 15 & 4 & 7 \\
\hline
\end{tabular}

Table4: The patients who accurately recognized as having Diabetic Retinopathy called Sensitivity. The patients who accurately recognized as not having Diabetic Retinopathy called as Specificity. The Sensitivity, Specificity as well as Overall Accuracy of each classes of diabetic retinopathy disorder of our proposed model is shown in below table.

\begin{tabular}{|l|l|l|l|l|l|}
\cline { 2 - 6 } \multicolumn{1}{c|}{} & $\begin{array}{l}\text { Normal } \\
=0\end{array}$ & $\begin{array}{l}\text { Mild } \\
\text { NPDR } \\
=1\end{array}$ & $\begin{array}{l}\text { Modera } \\
\text { te } \\
\text { NPDR } \\
=2\end{array}$ & $\begin{array}{l}\text { Severe } \\
\text { NPDR } \\
=3\end{array}$ & $\begin{array}{l}\text { PDR } \\
=4\end{array}$ \\
\hline $\begin{array}{l}\text { Sensitivity( } \\
\%)\end{array}$ & 99.44 & 72.97 & 67 & 21.05 & 24.13 \\
\hline $\begin{array}{l}\text { Specificity( } \\
\%)\end{array}$ & 97.83 & 91.76 & 90.18 & 93.93 & 99.10 \\
\hline $\begin{array}{l}\text { Accuracy( } \\
\%)\end{array}$ & 98.63 & 89.86 & 83.83 & 90.13 & 93.15 \\
\hline
\end{tabular}

Where sensitivity (SE), Specificity (SP), Accuracy (ACC) is given by below formula:

$$
\begin{gathered}
S E=\frac{T P}{T P+F N} S P=\frac{T N}{T N+F P} \\
A C C=\frac{T P+T N}{T P+T N+F P+F N}
\end{gathered}
$$

\section{$\mathrm{TP}, \mathrm{TN}, \mathrm{FP}, \mathrm{FN}$ is defined as shown in below:}

True Positive $(\mathrm{TP})=$ accurately recognized DR images.

True Negative $(\mathrm{TN})=$ accurately recognized Non-DR images.

False Positive (FP)=Number of Non-DR images are incorrectly recognized as DR images.

False Negative $(\mathrm{FN})=$ Number of DR images are incorrectly recognized as Non-DR Images.

Best Model is achieved at epoch 60. This can be seen in the below shownlearning curve.

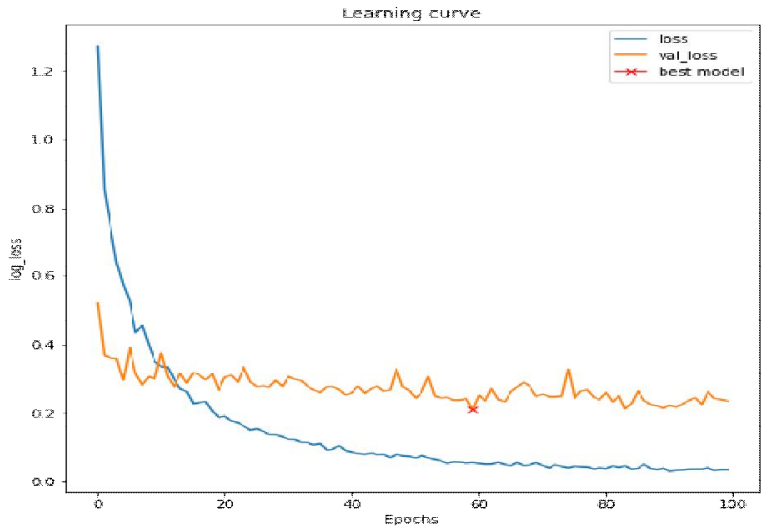

Figure 13: Learning curve showing best model at epoch 60 .

Accuracy of the model, validation data accuracy ,loss as well as validation loss is shown in below graphs. Vadation accuracy is used to check the perfomance of the model during training phase itself.

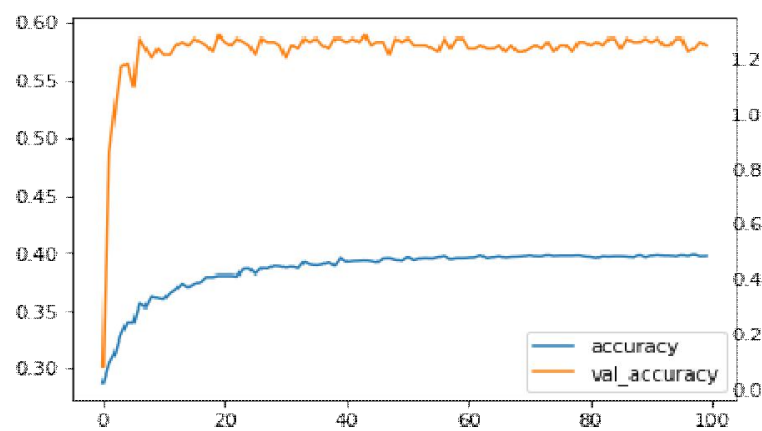

(a)

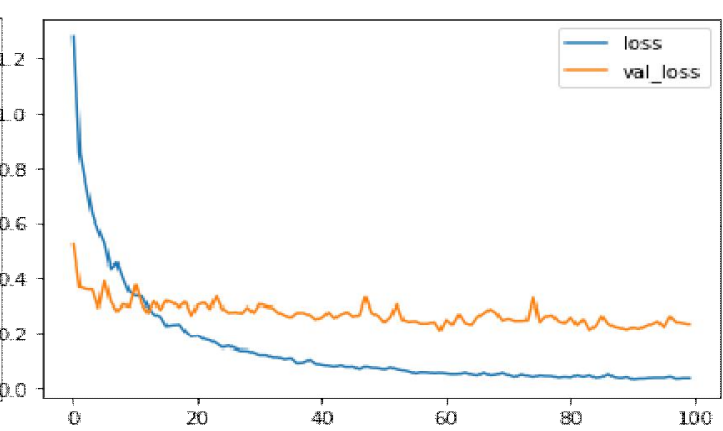

(b)

Figure14: (a) Graphical representation of epochs vs accuracy and validation accuracy. (b) Graphical representation of ephochs vs loss and validation loss. 
The functioning of the proposed model is evaluated using test data. Data file containing a test images as well as the csv file containing a images id of the patients is given as input to the proposed model in order to get the diagnosis of those images as outcome.

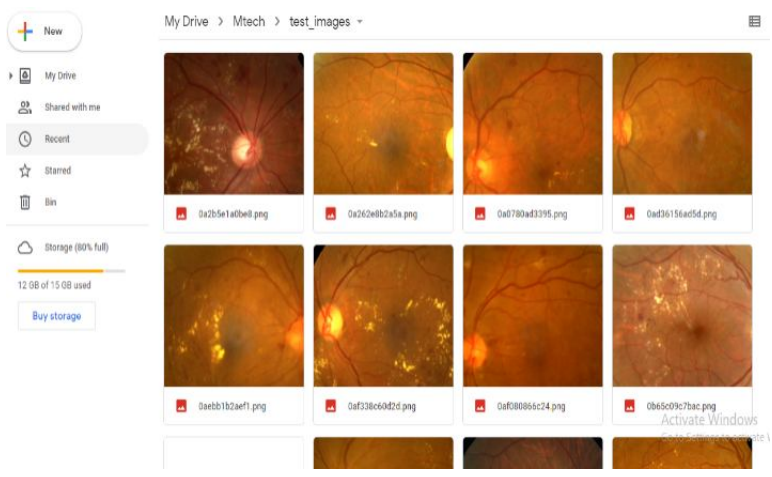

Figure15: Test images datafile containing 1928 image.

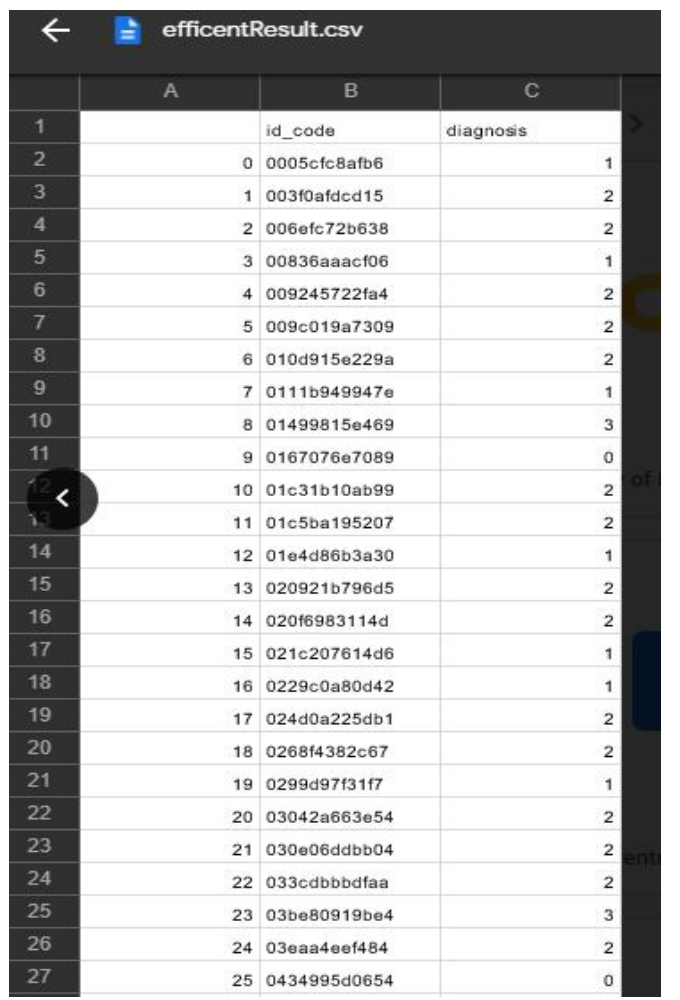

Figure16: The diagnosis outcome of the 1928 test images.

\section{CONCLUSION}

There are various supervised learning algorithms currently in use. But majority among them have need of pre-process or else post-process phases in order to determine distinct diabetic retinopathy disorder phases. Beside this, these prevailing algorithms, compulsory demanding hand-operated characteristic uprooting phases for the classification pertaining to fundus pictures. But our proposed methodology, deeply trained convolution neural network with EfficientNet B5 architecture is a healthful, beneficial model for each and every phases of diabetic retinopathy disorder. Hand-operated (manual) characteristic uprooting is not needed in this methodology. The proposed model can be saved and loaded when required. Simply giving csv or excel file containing image id of a patient and also the fundus image of that particular patient to the loaded model it gives an instant result of diagnosis for the given image. Thus it works in a real time manner.

\section{FUTURE WORK}

Future attempts can be established within many orientations. In order upgrade diabetic retinopathy classification precision (accuracy), a superior state-of-the-art convolution neural network grounded image categorization designs would be employed. For the purpose of helping diabetic retinopathy phase image identification, convolution neural network rooted object detection like veins, techniques would be supported. In order to achieve the implementation of more dense-structure diabetic retinopathy phase categorization, $\mathrm{CNN}$ grounded segmenting image like cotton wool spots [5] method would $n$ have inspected as well.

\section{REFERENCES}

[1] Nikhil M N; Angel Rose A," Diabetic Retinopathy Stage Classification using CNN", International Research Journal of Engineering and Technology (IRJET), vol.06, issue 05, May 2019, p-ISSN: 2395-0072.

[2] T Chandrakumar; R Kathirvel," Classifying Diabetic Retinopathy using Deep Learning Architecture", International Journal of Engineering Research \& Technology (IJERT), vol.5, issue 06, June 2016, ISSN: 2278-0181.

[3] Harry Pratt; FransCoenen; Deborah M Broadbent; Simon P Harding; Yalin Zheng, "Convolutional Neural Networks for Diabetic Retinopathy", International Conference On Medical Imaging Understanding and Analysis 2016, MIUA 2016, 6-8 July 2016, Loughborough, UK.

[4] Carson Lam; Darvin Yi; Margaret Guo; Tony Lindsey," Automated Detection of Diabetic Retinopathy using Deep Learning", Biomedical Informatics Department, Stanford University, Palo Alto, CA; School of Medicine, Stanford University, Palo Alto, CA; NASA Ames Research Center, Mountain View, CA.

[5] Xiaoliang Wang; Yongjin Lu; Yujuan Wang; Wei-Bang Chen," Diabetic Retinopathy Stage Classification using Convolutional Neural Networks", July 2018.

[6] ParshvaVora and Sudhir Shrestha," Detecting Diabetic Retinopathy Using Embedded Computer Vision”, October 2020.

[7] Parvathy EN and BharadwajaKumsr G," Diabetic Retinopathy Image Classification Using Deep Neural Network", AJPCR, February 2017.

[8] N. Cheung, G. Tikellis, and J. J. Wang, "Diabetic retinopathy," Ophthal-mology, vol. 114, no. 11, pp. 2098_2099, 2010.

[9] S. R. Flaxman et al., "Global causes of blindness and distance visionimpairment 1990_2020: A systematic review and meta-analysis," LancetGlobal Health, vol. 5, no. 12, pp. e1221_e1234, 2017. 
Vidya Manjunath Purushan et al., International Journal of Advanced Trends in Computer Science and Engineering, 10(3), May - June 2021, 1872 - 1882

[10] Mohamed Shaban; ZelihaOgur; Ali Mahmoud; Andrew Switala; Ahmed Shalaby; Hadil Abu Khalifeh; Mohammed Ghazal; LuayFraiwanID; GuruprasadGiridharan; Harpal Sandhu; Ayman S. El-BazID," A convolutional neural network for the screening and staging of diabetic retinopathy”, June 2020.

[11] Zhiguang Wang and Jianbo Yang, "Diabetic Retinopathy Detection via Deep Convolutional Networks for Discriminative Localization and Visual Explanation", 2018.
[12] Xianglongzeng; Haiquanchen; Yuan luo and Wenbin ye, "Automated Diabetic Retinopathy Detection Based on Binocular Siamese-Like Convolutional Neural Network", March 2019, IEEE. 